\title{
Gastrointestinal symptom severity in irritable bowel syndrome, inflammatory bowel disease and the general population
}

\author{
A. D. Lee Le, $2,4,5^{2}$ \\ B. M. Spiegel ${ }^{1,2,4,6,7}$ \\ R. D. Hays ${ }^{3,7,8}$ \\ G. Y. Melmed ${ }^{1}$ \\ R. Bolus ${ }^{4,6}$ \\ D. Khanna 9 \\ P. P. Khanna ${ }^{9}$ | L. Chang 6,10 \\ G.Y. Melmed
}

${ }^{1}$ Center for Outcomes Research and Education, Cedars-Sinai Medical Center, Los Angeles, CA, USA

${ }^{2}$ Department of Medicine, Division of Gastroenterology, VA Greater Los Angeles Healthcare System, Los Angeles, CA, USA

${ }^{3}$ RAND Health Program, Santa Monica, CA, USA

${ }^{4}$ UCLA/VA Center for Outcomes Research and Education (CORE), Los Angeles, CA, USA

${ }^{5}$ Division of Gastroenterology, Kaiser Permanente Los Angeles Medical Center, Los Angeles, CA, USA

${ }^{6}$ Department of Medicine, Division of Digestive Diseases, David Geffen School of Medicine at UCLA, Los Angeles, CA, USA

${ }^{7}$ Department of Medicine, UCLA Division of General Internal Medicine, Los Angeles, CA, USA

${ }^{8}$ Department of Health Services, UCLA Fielding School of Public Health, Los Angeles, CA, USA

${ }^{9}$ Division of Rheumatology, University of Michigan, Ann Arbor, MI, USA

${ }^{10} \mathrm{G}$. Oppenheimer Center of Neurobiology of Stress and Resilience, David Geffen School of Medicine at UCLA, Los Angeles, CA, USA

\section{Correspondence}

Lin Chang, G. Oppenheimer Center for Neurobiology of Stress and Resilience Los Angeles, CA, USA.

Email: linchang@ucla.edu

\begin{abstract}
Background: Irritable bowel syndrome (IBS) and inflammatory bowel disease (IBD) patients report similar gastrointestinal (GI) symptoms, yet comparisons of symptom severity between groups and with the general population (GP) are lacking.

Methods: We compared Patient-Reported Outcomes Measurement Information System (PROMIS ${ }^{\circledR}$ ) GI symptom scales measuring gastro-esophageal reflux (GER), disrupted swallowing, diarrhea, bowel incontinence, nausea/vomiting, constipation, belly pain, and gas/bloating in: (i) USA GP sample, (ii) IBS patients, and (iii) IBD patients from tertiary care and community populations. Symptom severity scores were based on T-score metric with mean $50 \pm 10$ (standard deviation) relative to the GP.

Key Results: Of 1643 patients enrolled, there were 253 IBS patients (68\% F, mean age $45 \pm 15$ years), 213 IBD patients ( $46 \% \mathrm{~F}$, mean age $41 \pm 14$ years), and $1177 \mathrm{GP}$ subjects ( $57 \% \mathrm{~F}$, mean age $46 \pm 16$ years). IBS patients reported greater severity of GER, disrupted swallowing, nausea/vomiting, belly pain, gas/bloating, and constipation symptoms than their IBD counterparts (all $P<.05$ ). Compared to the GP, IBD patients had worse belly pain, gas/bloating, diarrhea, and bowel incontinence, but less severe GER and disrupted swallowing (all $P<.05$ ), and IBS patients had more severe nausea/vomiting, belly pain, gas/bloating, and constipation (all $P<.05$ ). Women had more severe belly pain and gas/bloating than men, whereas men had more severe bowel incontinence (all $P<.05$ ).

Conclusion \& Inferences: IBS and IBD are associated with more severe GI symptoms compared to the GP excluding esophageal symptoms. Unlike IBD, IBS is not characterized by observable $\mathrm{Gl}$ inflammation but patients report more severe upper and lower $\mathrm{Gl}$ symptoms.
\end{abstract}

KEYWORDS

gender, inflammatory bowel disease, irritable bowel syndrome, symptom severity

\footnotetext{
Abbreviations: CD, Crohn's disease; GER, gastro-esophageal reflux; GI, gastrointestinal; GP, general population; HRQOL, health-related quality of life; IBD, inflammatory bowel disease; IBS, irritable bowel syndrome; PROMIS, Patient-Reported Outcomes Measurement Information System; UC, ulcerative colitis.

Funding information: This research was supported by NIH/NIAMS U01 AR057936A, the National Institutes of Health through the NIH Roadmap for Medical Research Grant (AR052177). Dinesh Khanna was also supported by. NIAMS K24 AR063120 Puja Khanna was supported by Ruth L. Kirschstein National Research Service Award (NRSA) Institutional Research Training Grant NIAMS 1 T32 AR053463 and ACR Research and Education Foundation Clinical Investigator Fellowship Award 2009_11. Ron D. Hays was also supported by NIH/NIA Grants P30-AG028748 and P30-AG021684, and NCMHD Grant 2P20MD000182. Lin Chang was also supported by NIDDK P50 DK64539.
} 


\section{1 | INTRODUCTION}

Irritable bowel syndrome (IBS) and inflammatory bowel disease (IBD) are gastrointestinal (GI) disorders that are associated with abdominal pain, alteration in bowel habits, relapsing-and-remitting courses, and psychological distress. ${ }^{1}$ In comparison to IBS in which disease severity is usually based on patient-reported symptoms, current research in IBD has focused on the use of serum, fecal, and colonic mucosal inflammatory biomarkers as surrogates for disease severity. ${ }^{2-4}$ Relatively less studied are patient-reported severity of Gl symptoms between these groups and the general population (GP).

IBS is a functional bowel disorder in which abdominal pain is associated with changes in bowel habits and disordered defecation. It occurs in $10 \%-20 \%$ of the general population and is more predominant in women and those with underlying psychological comorbidities or co-existing functional disorders. ${ }^{5-7}$ The etiology of IBS is multifactorial, but the pathogenesis is thought to be due to dysregulated brain-gut interactions in which peripheral and central sensitization can occur. Central sensitization at the spinal cord and brain level is associated with increased activation in brain regions involved in emotional arousal and pain modulation. ${ }^{8}$

Crohn's disease (CD) and ulcerative colitis (UC) are chronic immune-mediated disorders classified as IBDs that affect less than $1 \%$ of the USA population. ${ }^{9}$ Increased prevalence is seen in genetically predisposed individuals and certain ethnic groups. These diseases are thought be caused by chronic dysregulation of mucosal immune function, and therapies directed against suppression or modulation of inflammation are generally effective.

Although the extent to which these disease processes have overlapping pathologies is controversial, ${ }^{10}$ traditional thinking attributes the etiology of pain in IBD to objective inflammatory changes within the bowel and associated complications. It is commonly assumed that worsened symptom severity correlates with increased prevalence of inflammatory lesions and complications, however, this simplistic view of pain pathogenesis does not account for the fact that patients with IBS often will have similar complaints without objective disease pathology. While IBS and IBD have both been associated with worse general health-related quality of life (HRQOL), ${ }^{11}$ it remains unclear the extent that specific GI symptoms affect patients. Gl symptom questionnaires such as the Gastrointestinal Symptom Rating Scale (GSRS) and Quality of Life in Reflux and Dyspepsia (QOLRD), which measure the degree of Gl symptom discomfort, have been developed but have only been evaluated in patients with reflux disease and IBS and may not be applicable to a wider range of GI disorders and the GP. ${ }^{12-15}$

The NIH Patient-Reported Outcomes Measurement Information System (PROMIS ${ }^{\circledR}$ ) GI Symptom Scales assess gastro-esophageal reflux (GER), disrupted swallowing, diarrhea, bowel incontinence, nausea and vomiting, constipation, GI pain, and gas and bloating ${ }^{16}$ (Figure 1). The PROMIS ${ }^{\circledR} \mathrm{GI}$ instrument measures different facets of each symptom domain, including frequency, amount, bothersomeness, and impact. We previously found that the prevalence of heartburn was not different between a sample of the USA general population (GP) and a

\section{Key Points}

- Patients with irritable bowel syndrome (IBS) and inflammatory bowel disease (IBD) have similar gastrointestinal (GI) symptoms but studies comparing symptom severity between these groups and the general population are lacking.

- IBS patients experience worse upper and lower GI symptoms than IBD patients. Patients with either IBS or IBD experience worse epigastric and lower Gl symptoms than the general population, but comparable or less severe esophageal symptoms.

- Understanding the differences in symptom severity between patients will allow for improved symptom management.

patient group comprised of IBD and functional GI disorders, but regurgitation was more common in patients. ${ }^{17}$ In this study, we compare differences in upper and lower $\mathrm{Gl}$ symptom severity between patients with IBS, IBD, and the GP, adjusting for demographics. We also assess the effect of gender on Gl symptom severity. We hypothesize that the IBS and IBD patient groups will have greater symptom severity for belly pain and lower GI symptoms (e.g., diarrhea, constipation, bowel incontinence) than the GP, but that IBS patients will have greater severity of certain GI symptoms, including belly pain, gas and bloating, constipation than IBD patients, and the GP. In addition, we

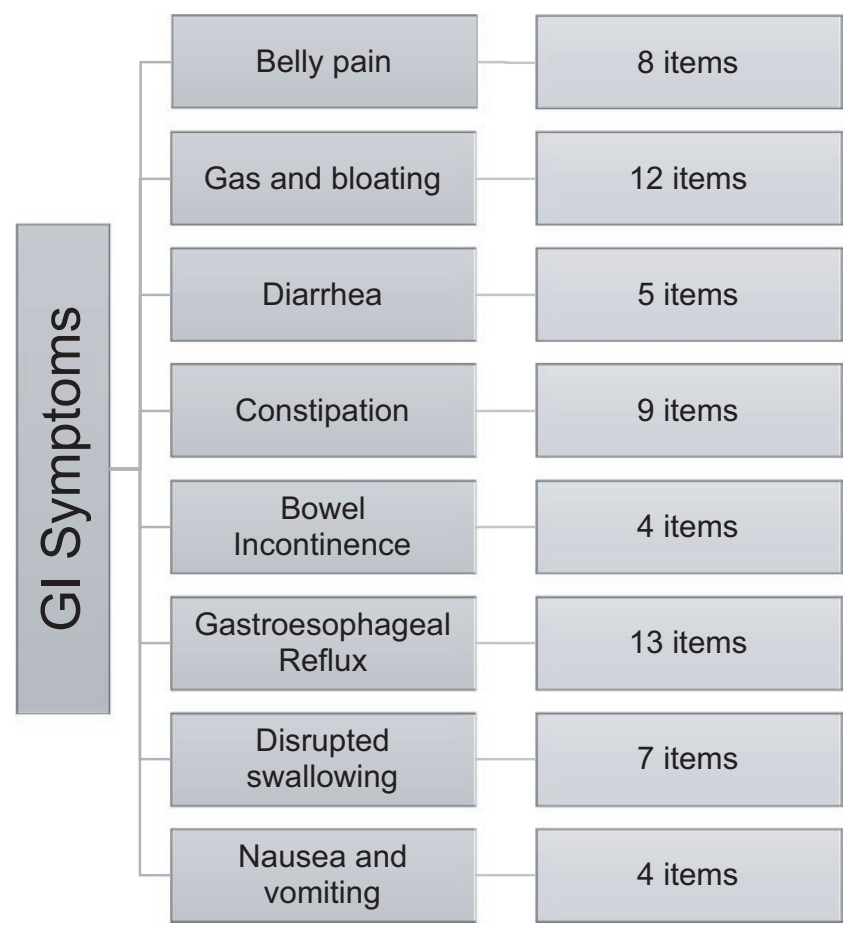

FIGURE 1 Gastrointestinal subdomains measured by the PROMIS ${ }^{\circledR}$ GI Symptom Scale. The eight symptom domains and the number of items in the GI PROMIS ${ }^{\circledR}$ questionnaire are shown 
hypothesize that women, in general, will report greater severity of belly pain and gas and bloating, as demonstrated in previous studies. ${ }^{18}$

\section{2 | METHODS}

\section{1 | Selection of participants}

This study recruited adult patients from a variety of academic and community-based gastroenterology clinics. Inclusion criteria for this cohort were as follows: (i) age 18 or older, (ii) able to speak, read, and understand English, (iii) have a physician-diagnosed GI disorder including IBS or IBD (CD or UC), and (iv) able to provide informed consent. Patients who did not meet all of these criteria were excluded from the study. Subjects included patients previously diagnosed with IBD seeking care at Cedars-Sinai Medical Center, a tertiary center in Los Angeles, and a specialty clinic at the University of Michigan, patients previously diagnosed with IBS seeking care at a functional bowel disorders clinic or responding to an advertisement for IBS research studies at the University of California Los Angeles, and patients with diverse GI conditions seeking care at a general GI clinic at the West Los Angeles Veterans Affairs Medical Center. Patients with IBS who were recruited from the clinics were previously diagnosed with IBS using the Rome III criteria ${ }^{5}$ by a physician with expertise in IBS before enrolling in this study. Patients with IBS who responded to a community advertisement completed a Rome III questionnaire and underwent a medical history and physical examination by a Gl physician to confirm the diagnosis of IBS. In addition, we partnered with the International Foundation for Functional Gastrointestinal Disorders (IFFGD) to survey a cohort of patients with diverse functional GI disorders (FGIDs) enrolled in IFFGD mailing lists.

All patients were invited to complete the confidential online survey instrument, administered using Survey Monkey software (http:// www.surveymonkey.com) and offered \$25 compensation. Patients without Internet access could request a paper survey be sent to their home, or completed in clinic, as needed. Patients were excluded from participation if they failed to provide informed consent, had a history of cognitive impairment that would interfere with participation, or if they had any concurrent medical or psychiatric condition that precluded participation.

An additional group of randomly selected participants designed to represent the GP in terms of gender, ethnicity, race, and education level based on the 2010 census data was recruited by a commercial survey research firm (CINT, http://www.cint.com) which administered the survey. Subjects were required to be 18 years of age or older and able to read English; there were no other exclusion criteria applied to the GP sample.

\section{2 | Instruments}

\subsection{1 | PROMIS ${ }^{\circledR}$ GI Symptom Scales}

The eight NIH PROMIS ${ }^{\circledR}$ GI symptom scales were developed to measure the breadth and depth of $\mathrm{Gl}$ symptoms experienced by individuals with a wide range of digestive disorders. Unlike diseasetargeted measures for specific patient populations, the PROMIS ${ }^{\circledR}$ GI symptom scales were designed for symptom assessment in all individuals, whether within the GP or patient groups, who are experiencing a Gl symptom. The Gl symptom scales were developed following the criteria for qualitative and quantitative development of $\mathrm{NIH}$ PROMIS ${ }^{\circledR}$ measures with oversight of the NIH PROMIS ${ }^{\circledR}$ Steering Committee. ${ }^{19-22}$ Details of the construction and evaluation of this instrument have been published. ${ }^{14}$ Factor analyses supported the creation of the eight symptom scales. Symptom severity was calculated from a scaled score in each category, estimated using a twoparameter graded response model with the mean set at 50 and the standard deviation to 10 relative to the USA GP with higher scores indicating more severe symptomatology.

\section{3 | Statistical analysis}

We calculated descriptive statistics for patients with IBS, IBD, and the GP. We performed bivariate analysis between groups to compare overall age, gender, ethnicity, education, marital status, and employment status using a $\chi^{2}$ test for categorical variables and a $t$ test for continuous variables. To compare Gl symptom domain differences between groups, we used analysis of covariance (ANCOVA) controlling for age, gender, race/ethnicity, education, and marital status, as these differed between groups and could affect symptom reporting. Main effects for gender were assessed within the pooled sample of GP, IBS, and IBD subjects. Significance was defined by a two-tailed $P<.05$.

\section{3 | RESULTS}

\section{1 | Clinical characteristics}

Overall, 1643 patients enrolled in this study. The IBS group consisted of 253 patients who met Rome III diagnostic criteria, confirmed by a physician, and had no evidence of organic disease. Among these patients, 28\% were classified as having IBS-constipation, 32\% IBS-diarrhea, $29 \%$ mixed, and $11 \%$ unknown based on Rome III criteria. ${ }^{5}$ The IBD group consisted of 213 patients, $10 \%$ were of IBD-unclassified subtype, whereas 33\% had Crohn's disease and 57\% had UC, which had previously been confirmed by accepted clinical, radiographic, endoscopic, and/or histologic criteria. Table 1 displays the number of patients recruited from each recruitment site. Table 2 lists the demographic information from the IBS, IBD, and general population subgroup ( $n=1177$ ). Significant differences between the IBS and IBD groups and the GP were found on age $(P=.008)$, female gender $(P=.005)$, race/ethnicity $(P<.0001)$, education level $(P<.001)$, marital status $(P=.016)$, and employment status $(P=.042)$.

\section{2 | Gl symptom differences between groups}

Table 3 shows the comparisons of symptom severity for each GI domain in patients with IBS or IBD to the GP. 


\begin{tabular}{lcccccc} 
& $\begin{array}{l}\text { Cedars Sinai } \\
\text { Medical } \\
\text { Center }\end{array}$ & UCLA & $\begin{array}{l}\text { VA Medical } \\
\text { Center West } \\
\text { Los Angeles }\end{array}$ & $\begin{array}{l}\text { University of } \\
\text { Michigan }\end{array}$ & $\begin{array}{l}\text { IFFGD } \\
\text { mailing } \\
\text { list }\end{array}$ & CINT \\
\hline IBS & 4 & 182 & 54 & 9 & 4 & - \\
IBD & 168 & 14 & 30 & 1 & 0 & - \\
\hline $\begin{array}{l}\text { General } \\
\text { Population }\end{array}$ & - & - & - & - & - & 1177 \\
\hline
\end{tabular}

TABLE 1 Recruitment of patients based on site

TABLE 2 Patient clinical characteristics

\begin{tabular}{|c|c|c|c|c|}
\hline & $\begin{array}{l}\text { Irritable } \\
\text { bowel } \\
\text { syndrome } \\
\text { (IBS, } n=253 \text { ) }\end{array}$ & $\begin{array}{l}\text { Inflammatory } \\
\text { bowel disease } \\
\text { (IBD, } n=213 \text { ) }\end{array}$ & $\begin{array}{l}\text { General } \\
\text { population (GP, } \\
n=1177 \text { ) }\end{array}$ & $P$ value \\
\hline Mean age $\pm S D$ (years) & $45 \pm 15$ & $41 \pm 14$ & $46 \pm 16$ & .008 \\
\hline Female gender (\%) & 68 & 46 & 57 & .005 \\
\hline \multicolumn{5}{|l|}{ Ethnicity/race (\%) } \\
\hline Caucasian & 56 & 58 & 73 & \multirow[t]{5}{*}{$<.0001$} \\
\hline Hispanic & 13 & 14 & 10 & \\
\hline African-American & 18 & 11 & 12 & \\
\hline Asian & 10 & 11 & 3 & \\
\hline Other & 2.1 & 6.8 & 1.6 & \\
\hline \multicolumn{5}{|l|}{ Education (\%) } \\
\hline Less than high school & 1.3 & 3.2 & 4.2 & \multirow[t]{4}{*}{$<.0001$} \\
\hline High school graduate & 33 & 36 & 60 & \\
\hline College graduate & 37 & 40 & 26 & \\
\hline Advanced degree & 29 & 21 & 9.9 & \\
\hline \multicolumn{5}{|l|}{ Marital status (\%) } \\
\hline Married & 39 & 42 & 45 & \multirow[t]{3}{*}{.016} \\
\hline Previously married & 23 & 15 & 22 & \\
\hline Never married & 38 & 43 & 33 & \\
\hline \multicolumn{5}{|l|}{ Employment (\%) } \\
\hline Employed/student & 71 & 72 & 65 & \multirow[t]{4}{*}{.042} \\
\hline Not working & 9.1 & 9.0 & 12 & \\
\hline Retired & 10 & 9.5 & 16 & \\
\hline Disabled & 9.5 & 10 & 7.5 & \\
\hline \multicolumn{5}{|l|}{ IBS subtype (\%) } \\
\hline IBS-constipation & 28 & & & \\
\hline IBS-diarrhea & 32 & & & \\
\hline IBS-mixed & 29 & & & \\
\hline Unknown & 11 & & & \\
\hline \multicolumn{5}{|l|}{ IBD subtype (\%) } \\
\hline Crohn's disease & & 33 & & \\
\hline Ulcerative colitis & & 57 & & \\
\hline Unclassified & & 10 & & \\
\hline
\end{tabular}

IBS vs GP. IBS patients reported significantly worse symptoms of belly pain $(P<.0001)$, nausea and vomiting $(P<.0001)$, gas and bloating $(P<.0001)$, diarrhea $(P<.0001)$, and constipation $(P<.0001)$ than the
GP. There were no significant differences between patients with IBS and the GP on GER, disrupted swallowing, and bowel incontinence (Figure 2). 
TABLE 3 Symptom domain analysis in irritable bowel syndrome and inflammatory bowel disease in comparison to the general population
FIGURE 2 Gastrointestinal symptom severity in irritable bowel syndrome (IBS) vs the general population. The symptom domain scores for the general population (GP, gray box) and the IBS patients (black box) are shown. The IBS patients had significantly higher scores (greater severity) for belly pain, gas/bloating, diarrhea, constipation, nausea, and vomiting compared to the GP (all $P<.05$, indicated by *)

\begin{tabular}{|llccc|}
\hline Symptom domain & Subgroup & Parameter & $P$ value & T statistic \\
\hline Gastro-esophageal reflux & IBS & 0.25 & .73 & 0.35 \\
& IBD & -4.9 & $<.0001$ & -6.5 \\
\hline Disrupted swallowing & IBS & 0.03 & .97 & 0.04 \\
\hline Nausea and vomiting & IBD & -4.1 & $<.0001$ & -5.4 \\
\hline Belly pain & IBS & 3.2 & $<.0001$ & 4.5 \\
& IBD & -1.1 & .14 & -1.5 \\
\hline Gas and bloating & IBS & 11.0 & $<.0001$ & 15 \\
\hline Diarrhea & IBD & 4.2 & $<.0001$ & 5.3 \\
\hline Bowel incontinence & IBS & 8.8 & $<.0001$ & 12 \\
& IBD & 2.7 & .0005 & 3.5 \\
\hline Constipation & IBS & 6.7 & $<.0001$ & 8.5 \\
& IBD & 6.9 & $<.0001$ & 8.7 \\
\hline & IBS & 1.9 & .12 & 2.5 \\
\hline
\end{tabular}

IBD, inflammatory bowel disease; IBS, irritable bowel syndrome.

Higher scores represent greater severity of symptoms. All comparisons controlled for age, gender, race/ethnicity, education, and marital status.

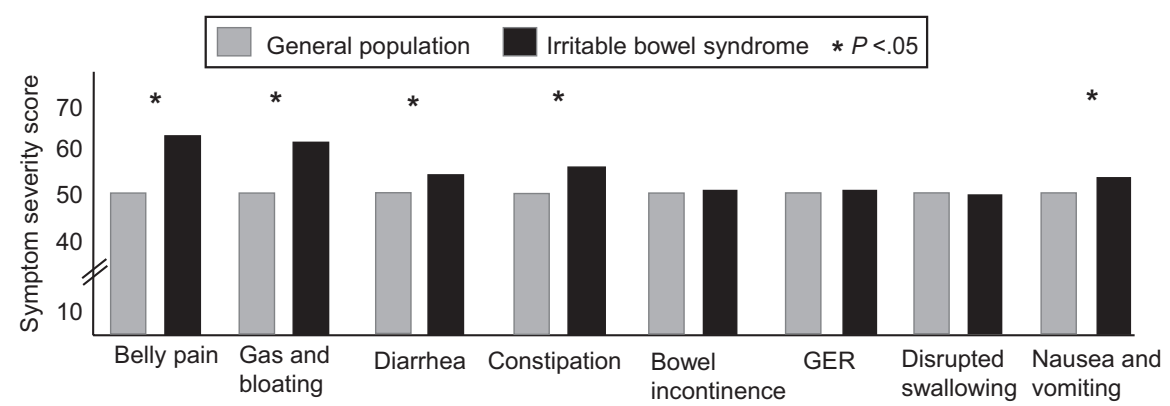

IBD vs GP. Patients with IBD had significantly worse belly pain $(P<.0001)$, gas and bloating $(P=.0005)$, diarrhea $(P<.0001)$, and bowel incontinence $(P=.004)$ than the GP. In contrast, patients with IBD experienced less severe symptoms of GER $(P<.0001)$ and disrupted swallowing $(P<.0001)$ than the GP. There were no significant differences in the severity of constipation and nausea and vomiting between these two groups (Figure 3).

IBS vs IBD. As shown in Figure 4, after controlling for age, gender, race/ethnicity, education, and marital status, most GI symptoms were reported as more severe by IBS patients than IBD patients: nausea and vomiting $(F=22, P<.0001)$, GER $(F=32, P<.0001)$, disrupted swallowing $(F=20, P<.0001)$, belly pain $(F=55, P<.0001)$, gas and bloating $(F=42, P<.0001)$, and constipation $(F=41, P<.0001)$. There were no significant differences in the severity of diarrhea $(F=0.06, P=.80)$ and bowel incontinence $(F=0.19, P=.69)$ between patients with IBS and IBD (Figure 4).

Main effects of gender. Three symptom domains differed significantly between men and women in the model. Women reported more severe belly pain $(P=.0026)$ and gas and bloating $(P<.0001)$, but had less severe bowel incontinence than men $(P<.0001)$. Although there was a trend for less severe disrupted swallowing in women vs men $(P=.054)$, there were no significant differences in nausea and vomiting, diarrhea, and constipation. There were no significant interactions between gender and group for any of the symptom domains.

\section{4 | DISCUSSION}

The PROMIS ${ }^{\circledR}$ instruments are patient-reported outcome measures that have been applied to a variety of diseases. ${ }^{23-26}$ Our study has been uniquely designed to allow comparisons of symptom severity in patients with IBS, IBD, and the GP, which have not previously been examined. Our main findings indicate that patients with IBS have more severe upper and lower $\mathrm{Gl}$ symptoms, excluding diarrhea and bowel incontinence, than patients with IBD. In addition, we found that patients with IBS and IBD report more severe epigastric and lower GI symptoms than the GP, but reported esophageal symptom severity that is comparable or less severe than the GP, respectively. Lastly, the 


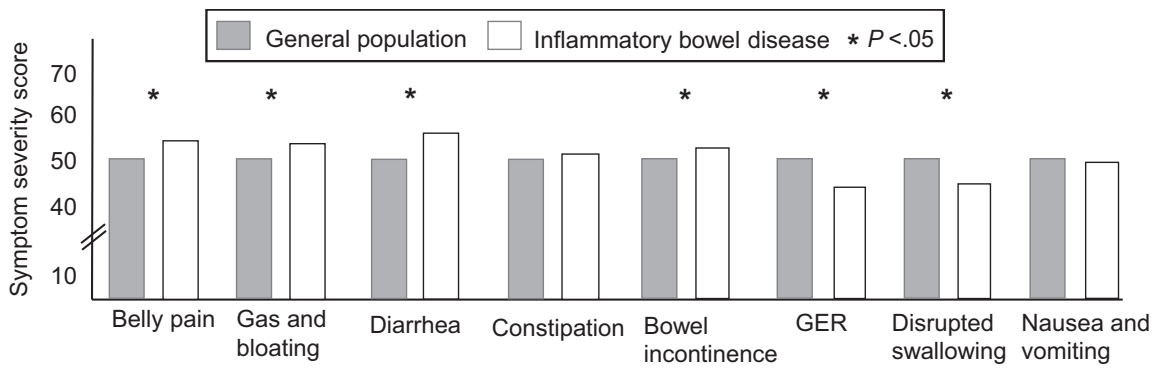

FIGURE 3 Gastrointestinal symptom severity in inflammatory bowel disease (IBD) vs the general population. The symptom domain scores for the general population (GP, gray box) and the IBD patients (white box) are shown. The IBD patients had significantly higher scores (greater severity) for belly pain, gas/bloating, diarrhea, bowel incontinence, but lower severity scores for gastro-esophageal reflux (GER) and difficulty swallowing compared to the GP (all $P<0.05$, indicated by*)

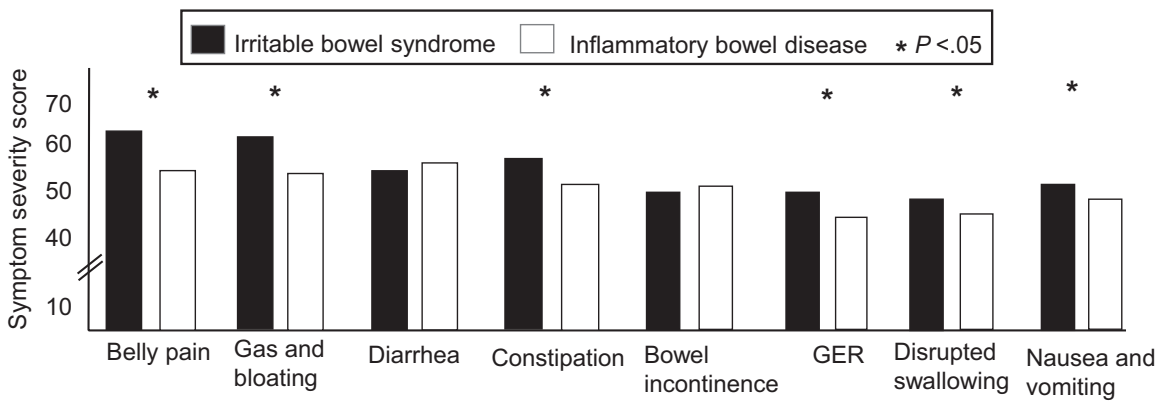

FIGURE 4 Gastrointestinal symptom severity in irritable bowel syndrome (IBS) vs inflammatory bowel disease (IBD). The symptom domain scores for the IBS patients (black box) and the IBD patients (white box) are shown. The IBS patients had significantly higher scores (greater severity) for belly pain, gas/bloating, constipation, gastro-esophageal reflux (GER), difficulty swallowing, and nausea and vomiting than the IBD patients (all $P<0.05$, indicated by *)

analysis of gender differences showed that overall, women reported more severe symptoms of gas and bloating and belly pain, but less severe symptoms of bowel incontinence and disrupted swallowing than men.

Patients with IBS reported more severe lower GI symptoms including belly pain, constipation, and gas and bloating than their IBD and GP counterparts. These results are consistent with a prior study performed three decades ago, showing that abdominal distention, straining at stool, and scybala were more likely in IBS than IBD. ${ }^{27}$ While bloating is a symptom that can often be seen in both conditions, it is a predominant symptom in patients with functional GI disorders particularly IBS and chronic constipation. ${ }^{28,29}$ In addition, IBS has a greater female predominance than IBD, and women, more so than men, report symptoms of gas, bloating, and constipation. ${ }^{30}$ Although our analysis is limited in that it generalizes symptoms in patients with IBS and IBD, without accounting for temporal variations in disease activity or the degree of IBD inflammation at the time of assessment, our results suggest that IBS patients, often with unremarkable endoscopic findings, have more severe symptoms than patients with IBD, a condition whereby disease pathology can be monitored by objective inflammatory and anatomic biomarkers. The comparable severity of diarrhea and bowel incontinence (as opposed to constipation) in IBS and IBD is not unexpected as diarrhea is a common feature of both $\mathrm{Gl}$ conditions.

The greater GI symptom severity seen in IBS patients is likely multifactorial. Patients' illness experience reflects upon how they perceive their sickness in the context of psychosocial and demographic conditions. ${ }^{31}$ IBS is a stress-sensitive disorder in which stress is associated with enhanced colonic motility and enhanced visceral perception. ${ }^{32,33}$ Hypervigilance, an increased attention to noxious stimuli, or an increased tendency to report sensations as bothersome has been demonstrated in IBS. ${ }^{34}$ In fact, patients with UC in remission with IBS symptoms were found to have worse Gl symptoms, psychological distress, and poorer physical and mental quality of life than patients with UC in remission without IBS. ${ }^{35}$ Although not directly examined in this study, these neurobiological and behavioral changes may explain why there is significantly greater severity of GI symptoms in IBS than IBD and the GP. Prior brain imaging studies have suggested that patients with IBS have increased activation of limbic and paralimbic circuits involved with emotional stress and pain, while patients with UC and healthy controls show an inhibition of these central pathways. ${ }^{36}$ This is supported clinically by the fact that IBD patients showing mild inflammation of their disease have rectal hyposensitivity (i.e., lower sensitivity) when undergoing rectal distention studies compared to IBS patients. ${ }^{37}$

We also found significant variation in upper GI symptom severity among IBS, IBD, and GP. Compared to the GP, IBD patients reported worse lower $\mathrm{Gl}$ symptoms, but significantly less severe esophageal symptoms than the GP. Although this is a novel finding, our analysis is limited in that we did not account for IBD disease phenotypes, subtypes, or disease location. It should also be noted that while Crohn's disease can affect the upper $\mathrm{Gl}$ tract, it is a rare phenomenon and only 
affects $0.5 \%-4 \%$ of patients with this disease ${ }^{38}$ and it is unlikely that active inflammation has significant contribution to upper Gl symptoms in IBD.

Prior studies have demonstrated that up to $30 \%-40 \%$ of patients with IBS will also report coexisting symptoms of GER, ${ }^{39,40}$ but the prevalence of IBD patients reporting GER symptoms has not been well studied. In the GP, the prevalence of GER ranges from $10 \%$ to $20 \%$ in Western populations. ${ }^{41} \mathrm{~A}$ possible explanation for the decreased upper GI symptom severity in IBD patients is that IBD is predominantly a disease that affects the distal bowel (ileum and colon) with rare involvement of the upper gastrointestinal tract, and in comparison, these patients may experience relatively less severe upper tract symptoms when contrasted to their severity of their lower GI symptoms. Evidence supporting this is based on prior studies demonstrating individuals distracted from pain will often report diminished pain severity. ${ }^{42,43}$ This view is consistent with the theory of attention-related modulation of pain processing in which attention allocated toward symptoms that are not specific to a particular pain will diminish the sensation of that pain. ${ }^{44} \mathrm{An}$ alternative explanation for these findings is that all of the surveyed patients with IBD and IBS were under the care of gastroenterologists who may have been adequately treating GER symptoms with antireflux measures. This may also explain why patients with IBS have comparable, rather than worse, GER symptoms than the GP. Of note, we previously found that a larger group of GI outpatients, which included our patients with IBS and IBD but also others with diverse $\mathrm{GI}$ conditions and systemic sclerosis, had worse regurgitation but similar heartburn symptoms than the GP using the PROMIS $^{\circledR}$ GI Symptom items. ${ }^{17}$ The prior inclusion of patients with gastro-esophageal reflux disease (GERD) and systemic sclerosis, which commonly coexists with GERD, with Gl symptoms in the previous study likely explains these differing results. Further studies should be performed to investigate these results in the context of medication usage, comorbidities, IBS and IBD disease phenotypes and subtypes, and IBD disease activity and location.

Women had more severe belly pain, gas, and bloating, and less severe disrupted swallowing and bowel incontinence than men. It is not surprising that women reported worse symptoms of gas and bloating and belly pain compared to men, as this has been previously shown in large cross-sectional population studies. ${ }^{18,45}$ Furthermore, these are the most common GI symptoms in IBS, and this group had a greater proportion of women than the other two groups in our study, particularly IBD. In addition, the finding that men tended to report worse symptom severity from disrupted swallowing has also been previously demonstrated and it is thought to be due to fact that women, when compared to men, have more preserved pharyngeal range of motion and cough reflex as they age, which contributes to a preserved ability to swallow. ${ }^{46,47}$ Interestingly, in the USA, bowel incontinence has been reported equally among genders, but may be more prevalent in younger women. ${ }^{48}$ The severity of symptoms between men and women has been shown to vary significantly depending on the composition of the fecal leakage. Population studies indicate that while women report more incontinence from liquid and solid stools, they have less severe incontinence from mucoid-type stools. ${ }^{49,50}$ Our finding that women report overall less severe symptoms from bowel incontinence than men is surprising, however, our study is limited in that we did not distinguishing between type of bowel incontinence. Further studies examining gender differences in bowel incontinence are needed.

Our study has limitations. We do not have information on the number of patients with IBD who also would concomitantly meet diagnostic criteria for IBS. A recent meta-analysis found that up to $42 \%$ of IBD patients can have IBS-like symptoms. ${ }^{51}$ However, this metaanalysis was limited because all but one study either did not use an objective scoring system to measure disease activity in IBD or did not clearly specify if the objective measures that were used to determine disease remission were obtained at the same time IBS symptoms were assessed. ${ }^{52}$ In addition, we found significant differences in our IBD and IBS populations with regards to age, gender, race/ethnicity, education level, marital status, and employment status which may reflect inherent demographic differences in these diseases or may be related to way these patients were recruited. However, our analyses controlled for these demographic differences. As many of these patients were recruited from specialty GI clinics, we cannot exclude the fact our findings may inherently have a selection bias. For example, IBD and IBS patients who visit their gastroenterologist in the outpatient setting may be more likely to have more severe disease activity and symptoms than those who do not regularly follow-up with a physician, although this was not measured in our study. Similarly, IBS patients who were recruited from a mailing list may be more hypervigilant and tend to report more severe symptoms, however, only a very small minority of patients were recruited in this manner. In the case of IBD, further studies that could stratify a patient's IBD symptoms based on inflammatory biomarker levels, such as C-reactive protein or calprotectin, would be useful to help confirm our findings. Another limitation is that we are not able to assess $\mathrm{Gl}$ symptom severity in the subgroups of IBS (e.g., IBS-D, IBS-C, IBS-M) and IBD (i.e., Crohn's disease, ulcerative colitis) or stratify our results by IBD disease activity, phenotype (e.g., Montreal or Vienna classification), and location due to the relatively small sample sizes.

In conclusion, using the PROMIS ${ }^{\circledR} \mathrm{GI}$ symptom scales, we found significant symptom severity differences between genders, IBS, IBD, and the GP. This study is unique in its ability to compare symptom perception in these disorders, and highlights the importance of understanding the impact of brain-gut interactions in Gl symptom assessment. Although most physicians will treat objective signs of IBD intestinal inflammation, IBS symptom severity may not primarily reflect macroscopic or microscopic inflammation, but may rather represent central amplification of viscerosensory input. In fact, symptom severity assessment is arguably more critical in the management of functional GI disorders like IBS where intestinal inflammation has not been consistently demonstrated. It is possible that the PROMIS ${ }^{\circledR} \mathrm{GI}$ symptom severity scales will correlate better with objective biologic markers than other severity instruments in IBS and IBD, but further studies are needed. Future studies should also include prospective assessment of symptom severity in well-characterized IBS and IBD patients to confirm our findings, and also determine if these symptom scales are responsive to treatment effects. 


\section{CONFLICTS OF INTEREST}

Brennan Spiegel has received grant support from AstraZeneca, Commonwealth Laboratories, GI Logic, Ironwood, Nestle Health Sciences, Shire, Takeda and has ownership in My Total Health and GI Logic. Dinesh Khanna has served as consultant and/or received grant support from Actelion, Astra-Zeneca, Bayer, BMS, DIGNA, Genentech, Gilead, InterMune, Merck, Takeda, Savient, and United Therapeutics. Puja P. Khanna has received grant support from Astra-Zeneca, and served as consultant for Takeda, Ironwood, Savient, Crealta, and Horizon. Ron D. Hays has served as a consultant to Amgen, Allergan, Pfizer, and the Critical Path Institute. Gil Melmed has served as a consultant for Abbvie, Celgene, Jannsen, Luitpold, Medtronic, Pfizer, RedHill Biopharma, Takeda, UCB and has received research support from Shire, Prometheus labs Jannsen, Celgene, Takeda, Gilead. Lin Chang has served on scientific advisory boards for Allergan, Ironwood, Synergy, IM Health care Science LLC, and Bioamerica. She also served as a speaker at a Takeda CME conference and Allergan symposium.

\section{AUTHOR CONTRIBUTION}

$\mathrm{AL}$ performed the research, analyzed and interpreted the data, and drafted the manuscript; BS participated in the design of the study, interpretation of the data, review and editing of manuscript, and funding; $\mathrm{RH}$ participated in the design of the study, statistical analysis, and interpretation of the data, review of the manuscript; GM participated in the design of the study and interpretation of the data, and review of the manuscript; RB participated statistical analysis and review of the manuscript; DK participated in the design of the study, interpretation of the data, review of the manuscript, and funding; PK participated in the design of the study and interpretation of the data, and review of the manuscript; LC participated in the design of the study, research, analyzed and interpreted the data, and contributed in writing the manuscript.

\section{ACKNOWLEDGMENTS}

We would like to thank Nancy and William Norton and the IFFGD for providing support and access to their patient population. We also would like to thank Sally Bolus and Jennifer Talley for their contributions in helping to manage the study.

\section{REFERENCES}

1. Pellissier S, Dantzer C, Canini F, Matheiu N, Bonaz B. Psychological adjustment and autonomic disturbances in inflammatory bowel disease and irritable bowel syndrome. Psychoneuroendocrinologly. 2010;35:653-662.

2. Keohane MB, O'Mahony C, O'Mahony L, O'Mahony S, Quigley EM, Shanahan F. Irritable bowel syndrome-type symptoms in patients with inflammatory bowel disease: a real association or reflection of occult inflammation? Am J Gastro. 2010;105:1789-1794.

3. Jelsness-Jorgensen LP, Bernklev T, Moum B. Calprotectin is a useful tool in distinguishing coexiting irritable bowel-like symptoms from that of occult inflammation among inflammatory bowel disease patients in remission. Gastroenterol Res Pract. 2013;2013:1-4.

4. Sydora MJ, Sydora BC, Fedorak RN. Validation of a point-of-care desk top device to quantitate fecal calprotectin and distinguish inflammatory bowel disease from irritable bowel-syndrome. J Crohns Colitis. 2012;2:207-214.

5. Longstreth GF, Thompson WG, Chey WD, Houghton LA, Mearin F, Spiller RC. Functional bowel disorders. Gastroenterology. 2006;130:1480-1491.

6. Lovell R, Ford A. Effect of gender on prevalence of irritable bowel syndrome in the community: systematic review and meta-analysis. Am J Gastro. 2012;107:991-1000.

7. Whitehead WE, Palsson O, Jones KR. Systematic review of the comorbidity of irritable bowel syndrome with other disorders: what are the causes and implications? Gastroenterology. 2002;122:1140-1156.

8. Larsson MB, Tillisch K, Walter SA. Brain responses to visceral stimuli reflect visceral sensitivity thresholds in patients with irritable bowel syndrome. Gastroenterology. 2012;142:463-472.

9. Loftus EV. Clinical epidemiology of inflammatory bowel disease: incidence, prevalence, and environmental influences. Gastroenterology. 2004;126:1504-1517.

10. Long MD, Drossman D. Inflammatory bowel disease, irritable bowel syndrome, or what?: a challenge to the functional-organic dichotomy. Am J Gastro. 2010;105:1796-1798.

11. Naliboff B, Kim SE, Bolus R, Bernstein CN, Mayer EA, Chang L. Gastrointestinal and psychological mediators of health-related quality of life in IBS and IBD: a structural equation modeling analysis. Am J Gastro. 2012;107:451-459.

12. Svedlund J, Sjodin I, Dotevall G. GSRS - a clinical rating scale for gastrointestinal symptoms in patients with irritable bowel syndrome and peptic ulcer disease. Dig Dis Sci. 1988;33:129-134.

13. Wiklund IK, Junghard O, Grace E, et al. Quality of life in reflux and dyspepsia patients. psychometric documentation of a new diseasespecific questionnaire (QOLRAD). Eur J Surg Suppl. 1998;583:41-49.

14. Revicki DA, Wood M, Wiklund I, Crawley J. Reliability and validity of the gastrointestinal symptoms rating scale in patients with gastroesophageal reflux disease. Qual Life Res. 1998;7:75-83.

15. Kulich KR, Madisch A, Pacini F, et al. Reliability and validity of the Gastrointestinal Symptom Rating Scale (GSRS) and Quality of Life in Reflux and Dyspepsia (QOLRAD) questionnaire in dyspepsia: a sixcountry study. Health Qual Life Outcomes. 2008;6:1-12.

16. Spiegel BM, Hays R, Bolus R, et al. Development of the NIH Patient Reported Outcome Measurement Information System (PROMIS ${ }^{\circledR}$ ) Gastrointestinal Symptom Scales. Am J Gastro. 2014;109:1804-1814.

17. Cohen E, Bolus R, Khanna D, et al. GERD symptoms in the general population: prevalence and severity versus care-seeking patients. Dig Dis Sci. 2014;59:2488-2496.

18. Sandler RS, Walter FS, Liberman JN, et al. Abdominal pain, bloating, diarrhea in the United States. Dig Dis Sci. 2000;45:1166-1171.

19. Cella D, Yount S, Rothrock N, et al. The Patient-Reported Outcomes Measurement Information System (PROMIS): progress of an $\mathrm{NIH}$ roadmap cooperative group during its first two years. Med Care. 2007;45:S3-S11.

20. Spiegel BM. Patient-reported outcomes in gastroenterology: clinical and research applications. J Neurogastroenterol Motil. 2013;19:137-148.

21. Khanna P, Agarwal N, Khanna D, et al. Development of an online library of patient reported outcome measures in gastroenterology: the GI-PRO database. Am J Gastroenterol. 2014;109:234-248.

22. DeWalt DA, Rothrock N, Yount S, Stone AA, on behalf of the PROMIS Cooperative Group. Evaluation of item candidates: the PROMIS qualitative item review. Med Care 2007; 45: S12-S21.

23. Bajaj JS, Thacker LR, Wade JB, et al. PROMIS computerized adaptive testing are dynamic instruments to measure heath-related quality of life in patients with cirrhosis. Aliment Pharmacol Ther. 2011;34:1123-1132. 
24. Hung M, Baumhauer JF, Latt JD, et al. Validation of PROMIS physical function computerized adaptive tests for orthopaedic foot and ankle outcome research. Clin Orthop Relat Res. 2013;471:3466-3474.

25. Broderick JE, Schneider S, Junghaenel DU, Schwartz JE, Stone AA. Validity and reliability of patient-reported outcomes measurement information system (PROMIS) instruments in osteoarthritis. Arthritis Care Res. 2013;65:1625-1633.

26. Khanna D, Krishnan E, Dewitt EM, Khanna P, Spiegel B, Hays RD. The future of measuring patient-reported outcomes in rheumatology. Arthritis Care Res. 2011;63:486-490.

27. Thompson G. Gastrointestinal symptoms in irritable bowel compared to peptic ulcer disease and inflammatory bowel disease. Gut. 1984;25:1089-1092.

28. Chang L, Lee OY, Naliboff B, Schmulson M, Mayer EA. Sensation of bloating and visible abdominal distention in patients with irritable bowel syndrome. Am J Gastro. 2001;96:3341-3347.

29. Johanson JF, Kralstein J. Chronic constipation: a survey of the patient perspective. Aliment Pharmacol Ther. 2007;25:599-608.

30. Adeyemo MA, Spiegel BM, Chang L. Meta-analysis: do irritable bowel symptoms vary between men and women? Aliment Pharmacol Ther. 2010;32:738-755.

31. Pierret J. The illness experience: state of knowledge and perspective for research. Sociol Health IIIn. 2003;25:4-22.

32. Chang L. The role of stress on physiologic responses and clinical symptoms in irritable bowel syndrome. Gastroenterology. 2011;140:761-765.

33. Mayer EA, Naliboff BN, Chang L, Coutinho SV. Stress and the Gastrointestinal Tract V. Stress and irritable bowel syndrome. Am J Physiol Gastrointest Liver Physiol. 2001;280:519-524.

34. Naliboff B, Munakata J, Fullerton S, et al. Evidence for two distinct perceptual alterations in irritable bowel syndrome. Gut. 1997;41:505-512.

35. Jonefjall B, Strid H, Ohman L, Svedlund J, Berstedt A, Simren M. Characterization of IBS-like symptoms in patients with ulcerative colitis in clinical remission. Neurogastroenterol Motil. 2013;25:756-e578.

36. Mayer EA, Berman S, Chang L, et al. Differences in brain responses to visceral pain between patients with irritable bowel syndrome and ulcerative colitis. Pain. 2005;115:398-409.

37. Chang L, Munakata J, Mayer EA, et al. Perceptual responses in patients with inflammatory and functional bowel disease. Gut. 2000;47:497-505.

38. Kefalas CH. Gastroduodenal Crohn's disease. Proc (Bayl Univ Med Cent). 2003:16:147-151.

39. Talley NJ, Dennis EH, Schettler-Duncan VA, Lacy BE, Olden KW, Crowell MD. Overlapping upper and lower gastrointestinal symptoms in irritable bowel syndrome patients with constipation or diarrhea. Am J Gastroenterol. 2003:98:2454-2459.
40. Lovell RM, Ford AC. Prevalence of gastro-esophageal reflux-type symptoms in individuals with irritable bowel syndrome in the community: a meta-analysis. Am J Gastroenterol. 2012;107:1793-1801.

41. Dent J, El-Serag HB, Wallander MA, Johansson S. Epidemiology of gastro-oesophageal reflux disease: a systematic review. Gut. 2005;54:710-717.

42. Miron D, Duncan GH, Bushnell MC. Effects of attention on the intensity and unpleasantness of thermal pain. Pain. 1989;39:345-352.

43. Rode S, Salkovskis PM, Jack T. An experimental study of attention, labeling and memory in people suffering from chronic pain. Pain. 2011;94:193-203.

44. Villemure CM, Bushnell C. Cognitive modulation of pain: how do attention and emotion influence pain processing? Pain. 2002;95: 195-199.

45. Oh YL, Mayer E, Schmulson M, Chang L, Naliboff B. Genderrelated differences in IBS symptoms. Am J Gastroenterol. 2001;96: 2184-2193.

46. Logemann JA, Pauloski BR, Rademaker AW, Kahrilas PJ. Oropharyngeal swallow in younger and older women: videofluoroscopic analysis. $J$ Speech Lang Hear Res. 2002;45:34-444.

47. Dicpinigaitis PV, Rauf K. The influence of gender on cough reflex sensitivity. Chest. 1998;113:1319-1321.

48. Whitehead WE, Borrud L, Goode PS, et al. Fecal incontinence in US adults: epidemiology and risk factors. Gastroenterology. 2009;137:512-517.

49. Quander CR, Morris MC, Melson J, Bienaias JL, Evans DA. Prevalence of and factors associated with fecal incontience in a large community study of older individuals. Am J of Gastro. 2005;100:905-909.

50. Teunissen T, Lagro-Janssen A, van den Bosch W, van den Hoogen $\mathrm{H}$. Prevalence of urinary, fecal and double incontinence in the elderly living at home. Int Urogynecol J. 2004;15:10-13.

51. Halpin S, Ford A. Prevalence of symptoms meeting criteria for irritable bowel syndrome in inflammatory bowel disease: systematic review and meta-analysis. Am J Gastro. 2012;107:1474-1482.

52. Quigley EM, Bernstein CN. Editorial: "Irritable Bowel Symptoms" in inflammatory bowel disease: diagnostic uncertainty meets pathological reality. Am J Gastro. 2012;107:1483-1485.

How to cite this article: Lee AD, Spiegel BM, Hays RD, et al. Gastrointestinal symptom severity in irritable bowel syndrome, inflammatory bowel disease, and the general population. Neurogastroenterol Motil. 2017;29:e13003. https://doi. org/10.1111/nmo.13003 